Gestión territorial y medio ambiente

\title{
La gobernanza ambiental: el estudio del capital social en las Áreas Naturales Protegidas
}

Environmental Governance: Studying the Social Capital in Protected Areas

A governança ambiental: o estudo do capital social nas Áreas Naturais Protegidas

\section{Erika Cruz Coria*}

Lilia Zizumbo Villarreal ${ }^{*}$

Nuchnudee Chaisatit ${ }^{* * *}$

Recibido: 2 de octubre de 2017

Aprobado: 25 de julio de 2018

Doi: http://dx.doi.org/10.12804/revistas.urosario.edu.co/territorios/a.6147

Para citar este artículo:

Cruz, C. E., Zizumbo, V. L., \& Chaisatit, N. (2019). La gobernanza ambiental: el estudio del capital social en las Áreas Naturales Protegidas. Territorios (40), 29-51. Doi: http://dx.doi.org/10.12804/revistas.urosario. edu.co/territorios/a.6147

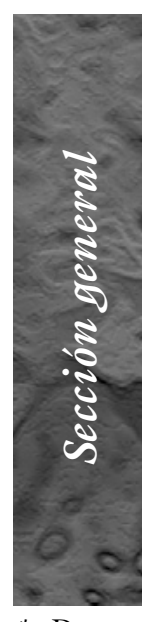

* Doctora en Ciencias Ambientales, Universidad Autónoma del Estado de México. Profesora-Investigadora, Instituto de Ciencias Económico Administrativas, Universidad Autónoma del Estado de Hidalgo. Correo electrónico: ecoria84@hotmail. com. ORCID: http://orcid. org/0000-0001-7984-0069

* Doctora en Sociología, Universidad Nacional de México. Profesora-Investigadora, Facultad de Turismo, Universidad Autónoma del Estado de México. Correo electrónico:lzv04@ yahoo.com. ORCID: https:// orcid.org/0000-00030639-5499

*** Maestra en Ciencias Ambientales, Universidad Autónoma del Estado de México. ProfesoraInvestigadora, Escuela de Turismo de la Universidad 
Palabras clave

Áreas Naturales Protegidas, capital social, actores sociales, estructura de relaciones, gobernanza ambiental.

Keywords

Protected Areas, social capital, social actors, relationship structure, environmental governance.

Palavras-chave

Áreas Naturais Protegidas, capital social, atores sociais, estrutura de relações, governança ambiental.

\section{territarias 40}

\section{RESUMEN}

Si bien, el Decreto de Áreas Naturales Protegidas (ANP) en la mayoría de los países de Centroamérica y el Caribe se ha dado en diferentes momentos históricos, esta figura jurídica ha surgido básicamente como un instrumento de conservación propuesta por la política de Estado; por tanto, las áreas se encuentran administradas por diversos organismos gubernamentales que a todas luces limitan la participación de otros actores sociales y el aprovechamiento sustentable de los recursos en estos territorios. Las ANP en México no son la excepción, estas se encuentran bajo un esquema de gobernabilidad que se caracteriza porque el Estado se erige como el actor central, el cual se encarga de normar tanto el acceso como el aprovechamiento de esos ecosistemas que también son objeto de interés por parte de múltiples actores sociales. En este contexto, el objetivo es proponer algunas herramientas teórico-metodológicas para la comprensión de la estructura de las relaciones sociales en las ANP mexicanas, lo cual, se supone, es fundamento para el diseño de intervenciones planificadas encaminadas al desarrollo de formas de gobernanza ambiental. Para ello, se retomó la perspectiva teórica del capital social (CS) con énfasis en el cs estructural, por su capacidad explicativa en relación con los vínculos de naturaleza horizontal y vertical, pero, sobre todo, es una herramienta que permite la comprensión de la gobernanza ambiental como un proceso socio-organizativo de carácter multiactoral.

\section{ABSTRACT}

In most Latin American countries, the decree of protected areas (PA) emerges as an instrument of nature conservation that is proposed by state policy. Therefore, the protected areas are under a management scheme carried out by the central government that limits the participation of other social actors and the sustainable use of resources in these territories. PA in Mexico are no exception, they are under a governance scheme in which the state stands as the central actor. Thus, this actor is responsible for regulating the access to and the use of these ecosystems that are of interest to multiple social actors. In this context, the objective is to propose some theoretical-methodological tools for understanding of the structure of social relations in the Mexican PA. This action is the basis for the design of planned interventions aimed at the development of environmental governance. For this paper, the theoretical perspective of social capital (SC) with emphasis on structural SC was used. This proposal has a broad capacity to explain the horizontal and vertical links in the whole structure of relationships. It allows the understanding of environmental governance as a multiactoral socio-organizational process.

\section{RESUMO}

Ainda que, o decreto de áreas naturais protegidas (ANP) na maioria dos países da Centro-América e o Caribe se tem dado em diferentes momentos históricos, esta figura jurídica tem surgido basicamente como um instrumento de conservação proposta pela política de Estado; portanto, as áreas se encontram administradas por diversos organismos governamentais que a todas luzes limitam a participação de outros atores sociais e o aproveitamento sustentável dos recursos nestes territórios. As ANP no México não são a exceção, estas encontram-se sob um esquema de governabilidade que se caracteriza porque o Estado erige-se como o ator central, o qual se encarrega de normatizar 
tanto o acesso como o aproveitamento desses ecossistemas que também são objeto de interesse por parte de múltiplos atores sociais. Neste contexto, o objetivo é propor algumas ferramentas teórico-metodológicas para a compreensão da estrutura das relações sociais nas ANP mexicanas, o qual se supõe é fundamento para o desenho de intervenções planificadas encaminhadas ao desenvolvimento de formas de governança ambiental. Para isso, retomou-se a perspectiva teórica do capital social (CS) com ênfase no cS estrutural, por sua capacidade explicativa em relação aos vínculos de natureza horizontal e vertical, mas especialmente, é uma ferramenta que permite a compreensão da governança ambiental como um processo socio-organizativo de carácter multiatoral.

\section{Introducción}

El Decreto de Áreas Naturales Protegidas (ANP) es promovido tanto por organismos internacionales como por los gobiernos nacionales como herramientas de política pública que impulsan la conservación y el funcionamiento armónico de estos ecosistemas. Desde el punto de vista ecológico, esta política de conservación ha demostrado ser eficaz (Selig \& Bruno, 2010); no obstante, en algunos casos, su creación ha reducido la problemática de la conservación al aislamiento de porciones de naturaleza intocada ignorando las relaciones económicas, sociales y culturales que estas guardan con las sociedades que las habitan (Toledo, 2005; Durand, Figueroa \& Trench, 2012).

$\mathrm{Al}$ menos en México, el Decreto de ANP forma parte del proyecto de adelgazamiento del Estado neoliberal y de la delegación de funciones a la ciudadanía; no obstante, en la práctica, surgen bajo esquemas de gobernabilidad que se caracterizan porque el Estado se erige como el actor central encargado de normar tanto el acceso como el aprovechamiento de estas áreas que, por su riqueza natural y cultural, se han convertido en espacios de interés público (Paz, 2008, Durand, 2014). A diferencia de lo que sucede en otros países como Estados Unidos y Canadá, en México estas áreas han sido decretadas en territorios donde confluyen una o más comunidades originarias y actores sociales a diferentes escalas y con diversos intereses; de ahí que el Estado ha tenido que crear espacios y canales de participación ${ }^{1}$ que le permitan interactuar con los actores sociales, pero siempre manteniendo su posición de "administrador" de los recursos, lo cual, a todas luces, limita la participación y la cooperación para la acción colectiva a favor de la conservación.

Los signos de deterioro ambiental que experimentan gran parte de estos ecosiste$\mathrm{mas}^{2}$ han conducido al impulso de algunas actividades económicas (turismo, servicios ambientales, forestería comunitaria, entre otros) que suponen ser ambientalmente compatibles con estas áreas, debido a que proveen los recursos financieros para un manejo más eficaz, al tiempo que motivan a los actores sociales a generar medidas adecuadas de conservación. Con dichas actividades, se han pretendido establecer alternativas para la conservación nico: nuchnudee@ucol. mx. ORCID: https://orcid. org/0000-0002-5577-0542

${ }^{1}$ En el caso de las ANP mexicanas, se han creado los Consejos Técnicos Asesores que, además de actuar como órganos de negociación de intereses entre la sociedad y el Estado, se han convertido en plataformas para la participación de los diferentes actores sociales en la toma de decisiones respecto a temas prioritarios como la conservación de la biodiversidad, sistemas productivos, equidad de género, etc. No obstante, desde su creación, estos consejos surgen como órganos reducidos a la mera posibilidad de emitir "sugerencias" para el manejo, conservación y aprovechamiento de los recursos naturales, por tanto, dan lugar a procesos de exclusión interna que refuerzan las inequidades al interior de estos espacios de uso múltiple-público (Peterson, 2011; Durand, Figueroa \& Trench, 2012).

${ }^{2}$ Entre los estudios más sobresalientes al respecto, está elrealizado por Arriola, Estrada, Ortega, Pérez \& Gijón (2014), se estudian 35 áreas naturales protegidas del centro de México y del Eje Volcánico Transmexicano, en las que se detectó 
$\Longrightarrow$

que los principales problemas son la explotación desmedida de los recursos naturales, cambio de uso de suelo, contaminación, introducción de especies exóticas e incendios.

3 Véanse Pérez, Zizumbo y Monterroso (2009); Esquivel, Cruz, Cadena y Zizumbo (2014); Cruz, Albrecht y Briones (2016) y Cruz, Zizumbo, Monterroso y Quintanilla (2013).

\section{territarias 40}

y el desarrollo sustentable; no obstante, muchas de estas han sido impulsadas predominantemente como una política de carácter sectorial, economicista y tomada “desde arriba" que ignora, entre otras cosas, la participación de los actores y la conformación de estructuras sociales (redes, organizaciones, vínculos) que faciliten la toma de decisiones y el aprovechamiento sustentable de los recursos.

Para algunos autores (Nenadovic \& Epstein, 2016; Blicharska, Orlikowska, Roberge \& Jurczak, 2016), los procesos exitosos de gobernanza ambiental en territorios multi-actores como las ANP mexicanas dependen, en gran medida, de la participación de los usuarios de los recursos naturales (gobiernos, empresas, comunidades, organizaciones no gubernamentales). La gobernanza ambiental es el "espacio" social donde se construyen las leyes, las políticas y los procedimientos formales en torno al aprovechamiento de los recursos naturales, por tanto, se relaciona con quiénes y cómo se toman las decisiones y con las características de los vínculos sociales que facilitan la cooperación y coordinación de quienes participan en estas acciones. En relación con esto, algunos estudios (Glimour, Dwyer \& Day, 2011; Marín, Gelcich, Castilla \& Berkes, 2012) reconocen al capital social (CS) como el factor que más influencia ejerce en la construcción y fortalecimiento de las capacidades socioorganizativas, que pueden incrementar la probabilidad de una gestión colectiva y sustentable desde el punto de vista de los actores y de sus prácticas.
Dicho lo anterior, el objetivo de este trabajo es proponer algunas herramientas teórico-metodológicas para la comprensión de la estructura de las relaciones sociales en las ANP mexicanas, lo cual se supone es fundamento para el diseño de intervenciones planificadas encaminadas al desarrollo de formas de gobernanza ambiental. Para ello, se retoma la perspectiva teórica del CS con énfasis en el CS estructural (CSE), por su capacidad explicativa en relación con los vínculos de naturaleza horizontal, pero, sobre todo, es una herramienta metodológica que permite el acercamiento a las relaciones entre los actores en los distintos niveles de la estructura social.

La reflexión que se presenta se desprende de una serie de observaciones realizadas por los autores en diversas ANP del sur y centro de México $^{3}$; no obstante, la intención no es establecer generalizaciones a partir del referente empírico, sino argumentar en torno a las herramientas teórico-metodológicas que pueden contribuir a la comprensión de la naturaleza de las relaciones entre los actores sociales que participan en la gestión de las ANP. Por tanto, metodológicamente este trabajo responde, por un lado, a un proceso exhaustivo de revisión bibliográfica $\mathrm{y}$, por el otro, a un desarrollo de tipo inductivo donde el insumo principal fue la evidencia empírica obtenida en trabajos previos, a partir de la cual se identificaron las categorías teóricas y metodológicas que se presentan en este trabajo.

En el primer apartado se aborda la existencia del Cs en la estructura de las 
relaciones como una condición necesaria para la conformación de formas de gobernanza ambiental. En el segundo, se señalan algunas de las problemáticas más importantes en la gestión centrando las argumentaciones en torno a la presencia de múltiples actores en las ANP mexicanas. Finalmente, en el tercer apartado se reflexiona sobre la importancia del diagnóstico del Cs, particularmente, del CSE como herramienta de análisis que permite visualizar la estructura de relaciones en términos de redes sociales, de las cuales emana este recurso como requisito indispensable en los procesos de gobernanza ambiental.

\section{Gobernanza ambiental y capital social}

De acuerdo con Schejtman \& Berdegué (2004), el enfoque territorial del Desarrollo Rural (ETDR) es "un proceso de transformación productiva e institucional en un espacio rural determinado, cuyo fin es reducir la pobreza rural" (p. 4). Esta propuesta tiene, por un lado, una naturaleza estrictamente económica que se refiere a la capacidad de los agentes y del tejido empresarial local para articular la economía del territorio. Y, por el otro, una dimensión extraeconómica que aborda los valores e instituciones locales que se van fortaleciendo en el ejercicio de los procesos de desarrollo.

Este último planteamiento apuesta por la transformación institucional como la emergencia de nuevos actores sociales capaces de generar formas alternativas de participación en la toma de decisiones y de intervención sobre los recursos del territorio (Rist, Chidambaranathan, Escobar, Wiesmann \& Zimmermann, 2007; Abbott, Green \& Keohane, 2016); es decir, propone una transformación en la forma de dirigir el desarrollo - alejado del control jerárquico y la centralidad del Estado-, caracterizado por un mayor grado de $\mathrm{CoO}^{-}$ peración e interacción entre los actores públicos, privados y sociales.

La transformación institucional se encuentra estrechamente ligada a procesos como la gobernanza, entendida no solo como un cambio en el sentido del gobierno sino como un proceso de participación que surge de "abajo hacia arriba" (Sánchez, Gallardo \& Ceña, 2014; Ojo \& Mellouli, 2016), en el cual intervienen los gobiernos en sus distintos niveles, pero también otras partes interesadas que pertenecen a la sociedad civil.

En este sentido, la gobernanza es un elemento esencial de transformación institucional para el desarrollo, sobre todo, en aquellos territorios donde la gestión de los recursos se hace más compleja a medida que convergen diversos actores sociales y se combinan la propiedad estatal, la propiedad común y la propiedad privada de la tierra (Ostrom, 1990; Paavola \& Adger, 2005); un ejemplo claro son la gran mayoría de las Áreas Naturales Protegidas (ANP) en países como México.

En la gestión de las ANP, la gobernanza ambiental se refiere a la facultad real de los actores sociales que interactúan en un territorio para controlar el acceso y el uso territarias 40

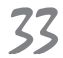


de los recursos naturales y, a su capacidad de ejercer influencia en los procesos de producción y consumo a partir de su aprovechamiento (Brenner, 2010; Stoll-Kleemann, et al., 2006). En décadas recientes, estos territorios han mostrado signos claros de deterioro provocados por la pobreza y la marginación de las poblaciones que las habitan; sin dejar de mencionar las políticas de desarrollo carentes de planificación y la reproducción de estructuras paternalistas que limitan la participación de los diferentes actores sociales en la gestión sustentable de los recursos naturales.

Si bien, el origen de estas problemáticas puede ser variada, lo cierto es que el debilitamiento institucional, económico, técnico y, sobre todo, organizacional y de las redes entre los actores sociales ha puesto en riesgo la conservación de los recursos naturales y las posibilidades de desarrollo de quienes los aprovechan. En este contexto, se hace cada vez más necesario el fortalecimiento de las capacidades socio-organizativas de los diversos actores involucrados, a fin de contribuir a la construcción de la gobernanza ambiental en dichos territorios.

De acuerdo con algunos autores (Bowles \& Gintis, 2002; Natera 2001, 2005; Nunkoo, 2017; Triyanti, Bavinck, Gupta \& Marfai, 2017), esas capacidades suelen sintetizarse en la noción de Cs; dicha categoría analítica es un ingrediente estructural que aumenta la probabilidad de que surjan capacidades sociales de acción colectiva en los territorios donde el Estado guarda una posición dominante en la toma de decisiones. La gobernanza ambiental requiere de ese cúmulo de virtudes cívicas y sociales disponibles en una sociedad que la hacen capaz de promover la participación de los actores sociales y la acción colectiva en los procesos sistémicos de desarrollo.

\section{La gestión turística de las Áreas Naturales Protegidas en México}

En México se han decretado 182 áreas naturales de carácter federal bajo distintas modalidades que, en conjunto, representan el 10,6\% del territorio nacional continental y el $22,05 \%$ de la superficie marina del país (CONANP, 2018). La Comisión Nacional de Áreas Naturales Protegidas (CONANP) es el órgano público encargado de la administración de estos ecosistemas; no obstante, cuando se trata de acciones de desarrollo in situ, se hacen presentes otros actores sociales, entre ellos: (i) las comunidades locales, (ii) cooperativas y/o empresas privadas, (iii) instituciones gubernamentales, (iv) instituciones académicas. La presencia de esta amplia diversidad de actores sociales convierte a las ANP en lo que Leeuwis \& Van den Ban (2004) denominan como “espacios de uso múltiple-público" (p. 18).

Particularmente, desde la perspectiva socio-organizativa, hay varias deficiencias y aspectos que no han sido suficientemente desarrollados en relación con la gestión de las ANP mexicanas (Paré \& Lazos, 2003; Brenner, 2010; Brenner \& De la Vega, 2014). La referencia empírica indica que gran parte de estas problemáticas deviene 
de la coincidencia de estos territorios con el de poblaciones campesinas e indígenas, por tanto, las instituciones no solo tienen que hacer frente a la variabilidad ecológica de estos ecosistemas sino también a la variedad cultural, a la cual se suman los altos índices de pobreza y marginación, entre otros problemas relacionados con la explotación de los recursos. Bajo este contexto y con dichas problemáticas, la participación social y la acción colectiva para la construcción de la gobernanza ambiental parece algo menor.

Si bien, la CONANP (2018) ha señalado que el $90 \%$ de las áreas decretadas cuentan con acciones de fortalecimiento de la participación social e institucional; lo cierto es que las contradicciones entre los objetivos de conservación de los recursos y los de desarrollo de las poblaciones son una constante que ha desencadenado un sin número de conflictos entre actores gubernamentales y sociales; por supuesto, sin dejar de mencionar las confrontaciones existentes al interior y entre las poblaciones que las habitan por temas relacionados con la tenencia de la tierra o los límites territoriales, por mencionar algunos.

La falta de alternativas económicas sustentables capaces de elevar la calidad de vida de la gente y de responder a las necesidades de conservación de estas áreas es otro de los aspectos que limitan la participación y la acción colectiva social a favor del desarrollo económico y social en estos territorios. En los casos donde se han logrado implementar algunas alternativas "ambientalmente compatibles" con el entorno (turismo, servicios ambientales, explotación forestal, entre otros), generalmente se han desarrollado ambientes sociales de mayor conflictividad. Pues, a la agenda de conflictos se han sumado las opiniones, intereses y acciones de otros actores sociales (instancias gubernamentales y privadas) que, ante el valor económico adquirido por los recursos naturales, intentan vincularse a las poblaciones locales para participar en la gestión y, en su caso, en el aprovechamiento de los recursos naturales de estos territorios (Azuela \& Mussetta, 2009; Brenner, 2010, Tejeda, 2014).

Algunos autores (Paré \& Lazos, 2003; Brenner, 2010) sugieren la elaboración de estudios de base que den luz sobre las dinámicas relacionales entre los actores sociales en estos territorios, a fin de visualizar las estructuras de control, los grupos marginados de los procesos en la toma de decisiones, el empoderamiento, entre otros aspectos que limitan o favorecen la toma de decisiones y la conformación de estructuras para el desarrollo de procesos de gobernanza ambiental a favor de la conservación de estos territorios.

En el caso de México, las instituciones encargadas de la gestión de las ANP han omitido el estudio de la dinámica relacional en términos de redes y sinergias de todo orden como requisito para la implementación de proyectos de desarrollo económico y social. La política pública y los programas de impulso al desarrollo han ignorado la morfología de las relaciones en el territorio, el rol de los actores, así como la naturaleza (negociación, subordinación, territarias 40

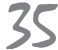


4 Grimble \& Wellard (1999) definen a los actores sociales como "any group of people, organized or unorganized, who share a common interest or stake in a particular issue or system [...] who can be at any level or position in society, from global, national and regional concerns down to the level of household or intrahousebold, and be groups of any size or aggregation" (p. 176).

\section{territarias 40}

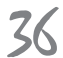

conflicto) y la intensidad de las relaciones (Lugo, 2011), la simetría o asimetría con la que estas se presentan y la transferencia de conocimiento como herramientas para enfrentar los retos en cuanto a la legitimidad, transparencia, rendición de cuentas, desarrollo y conservación en las ANP.

Este trabajo de investigación retoma la utilidad del diagnóstico como la vía para el estudio de las estructuras de las relaciones sociales en las ANP mexicanas. La reflexión que se presenta no está propiamente encaminada al abordaje de un marco metodológico de planificación participativa; pero sí propone una reflexión en relación con los elementos teórico metodológicos que pueden apoyar en la identificación de los recursos sociales que estimulan la vinculación de los actores sociales - a nivel micro, meso y macro- en estos territorios, lo anterior resulta fundamental en la generación de estructuras de gobernanza ambiental en términos de participación social.

A pesar de la abrumadora bibliografía en torno al estudio de la conservación, son pocos los documentos en términos metodológicos que son capaces de orientar sobre la comprensión de la estructura de las relaciones en ámbitos tan complejos como las ANP. En este caso, se retoma la perspectiva teórica del cs y, particularmente, la del CSE por su capacidad para dar explicación a la naturaleza de las relaciones en el plano del actor individual y, a su vez, a la dinámica de la estructura de las relaciones en su conjunto. Para Coleman (1990), el cs es una "herramienta" que permite realizar transiciones exitosas del nivel micro al macro social a partir de la explicación de los vínculos "verticales" y "horizontales".

Las contradicciones que se derivan del desarrollo social en las ANP mexicanas han abierto espacios de reflexión para la generación de metodologías integradas que, desde un enfoque territorial, contemplen la naturaleza de las relaciones entre los actores sociales como información fundamental para determinar las oportunidades de desarrollo de estas áreas de conservación. Es necesario el despliegue de reflexiones y propuestas metodológicas que no solo visualicen los recursos naturales y culturales comprometidos en el desarrollo sino también orienten sobre las características y capacidades de los agentes locales que participan en este proceso.

Para el caso mexicano, se reflexiona sobre el tipo de actores y recursos sociales que pueden incentivar la conformación de estructuras sociales con capacidad para transitar de una gestión de los recursos naturales basadas en estructuras de gobernabilidad a otras fundamentadas en procesos de gobernanza ambiental.

\section{La dinámica relacional en las ANP mexicanas}

\subsection{Mapeo de los actores sociales}

Los actores sociales que convergen en las ANP desempeñan un papel crítico en la gobernanza ambiental de estos territorios $^{4}$. El diagnóstico social de las ANP debe incorporar el "mapeo" de los agentes 
involucrados que están directa e indirectamente en la gestión de estos territorios. Lo anterior tiene implicaciones interesantes, pues da cuenta de la naturaleza (local, estatal, nacional) de los actores sociales y su incidencia sobre el territorio, de las lógicas de acción predominantes (individual/colectiva) y provee de un panorama superficial de la dinámica relacional (conflictos, alianzas, relaciones de cooperación, etc.) presente en estos espacios sociales.
Se observa que los actores sociales en las ANP operan en diferentes niveles de la estructura de las relaciones, su mapeo puede darse en un nivel micro que incluye las relaciones de los individuos en sus grupos primarios (familia, integrantes de la comunidad); en un nivel meso donde se encuentran aquellos grupos, redes sociales $\mathrm{o}$ asociaciones que trascienden el ámbito comunitario (Moyano, 2008; Brenner, 2010). Y, finalmente, las relaciones que

Figura 1. Distribución de las ANP federales en el territorio mexicano

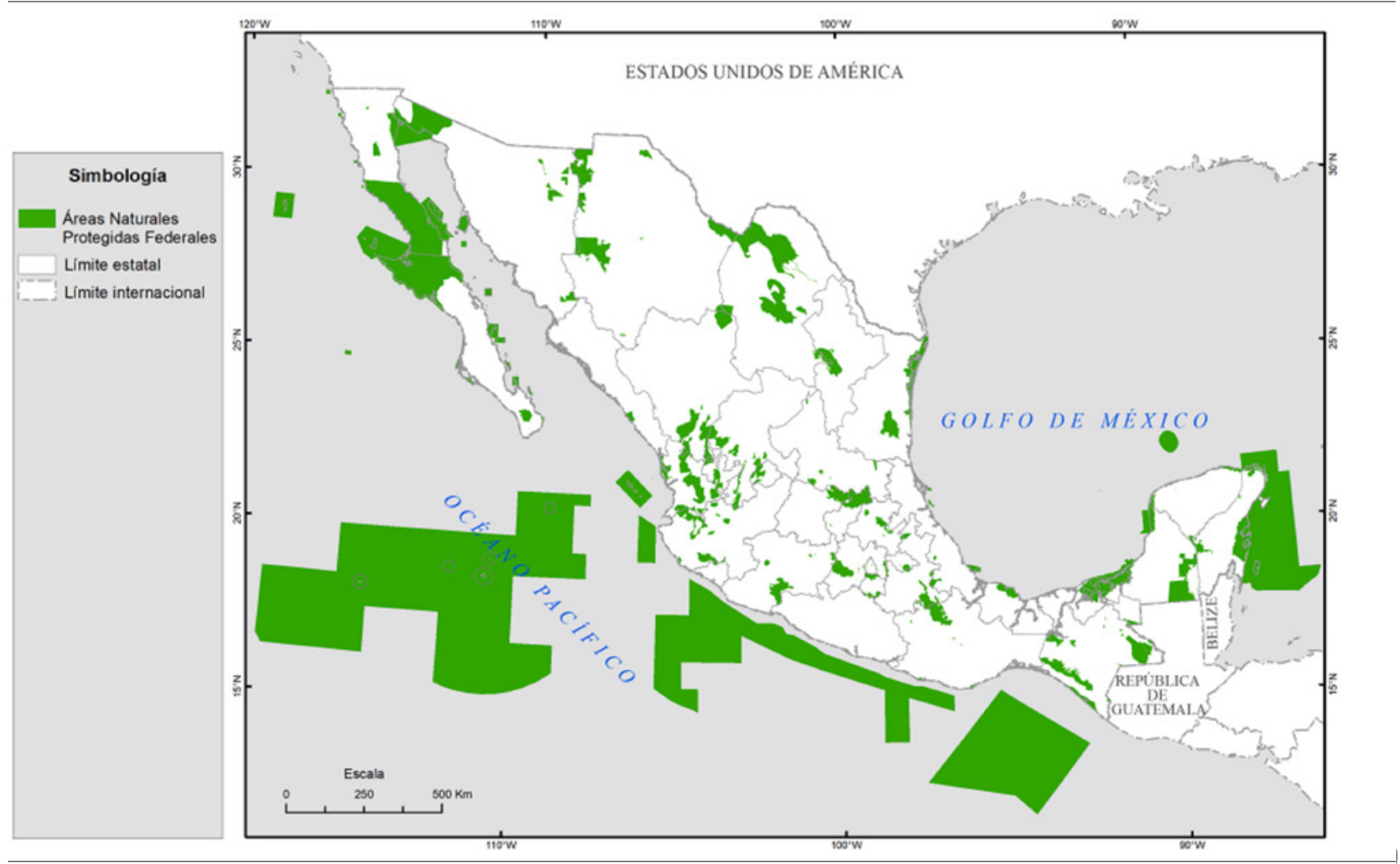

Fuente: Comisión Nacional de Áreas Naturales Protegidas, 2017. 
se establecen en el nivel macro, las cuales comprenden la cooperación entre instituciones públicas o privadas (figura 1 ).

Además de su ámbito de operatividad, también pueden ser clasificados en función de su acción sobre el territorio. Es vital la identificación de los actores a nivel micromeso, debido a que su acción "local" es amplia y tienen mayor influencia sobre los procesos económicos, políticos y sociales (Alonso, 2004). No obstante, el mapeo de los actores sociales no debe limitarse a los que tienen presencia material en el territorio; por el contrario, se debe contemplar aquellos cuya influencia no solo es local, sino también regional, nacional e internacional y que, aunque se reproducen en otros ámbitos, inciden activamente en la toma de decisiones a nivel local.

El ámbito de acción de los actores sociales se encuentra vinculado, a su vez, con una lógica de acción específica, la cual es indicador de la probabilidad de establecer acciones individuales/colectivas y estructuras de gobernanza incluyentes/ excluyentes que beneficien o perjudiquen a los interesados (figura 2). Así, cuando dominan los intereses personales sobre el aprovechamiento de los recursos naturales se está frente actores locales que actúan bajo una lógica individual, lo cual deriva en diversas formas de apropiación y control de los recursos. De acuerdo con algunos autores (Alonso, 2004; Beritelli, 2011), el dominio de la lógica individual debilita las posibilidades de establecer redes de participación y de toma de decisiones colectivas, lo que propicia el surgimiento de diferentes formas de "clientelismo", de dominación y control sobre los recursos naturales. En cambio, cuando la acción de los individuos funciona a partir de aspectos como la solidaridad, la toma de decisiones en conjunto y la construcción de objetivos en común, se está frente a una lógica colectiva (Valencia, 1990; Melucci, 1996).

Los actores locales también se encuentran vinculados con una lógica pública, que en términos no jurídicos se refiere a aquella que tiende a privilegiar el acceso abierto y visible de los recursos para todos (Rabotnikof, 2008); de alguna forma esta lógica remite a la acción del Estado. Aunque en un Estado capitalista como el que impera actualmente, las reglas en torno al aprovechamiento de lo "público" generalmente sirven a aquellos que tienen poder para imponerlas.

En el mapeo de los actores, lo importante es visualizar quiénes son los actores sociales y cuál el ámbito y lógica de acción predominante en relación con su participación en la gestión de las áreas. En el aprovechamiento de las ANP mexicanas, los actores que privilegian la esfera de los intereses individuales son las micro, pequeñas, medianas y grandes empresas que prestan servicios con fines comerciales, principalmente, a partir de actividades como el turismo o la forestería. Existen otro tipo de organizaciones que no actúan propiamente bajo una lógica individual ni empresarial. Por ejemplo, las organizaciones ejidales y comunales o las cooperativas de producción primaria que, ante la crisis agrícola, han adaptado sus estructuras 
Figura 2. Ejemplo de la estructuras de las relaciones en un ANP

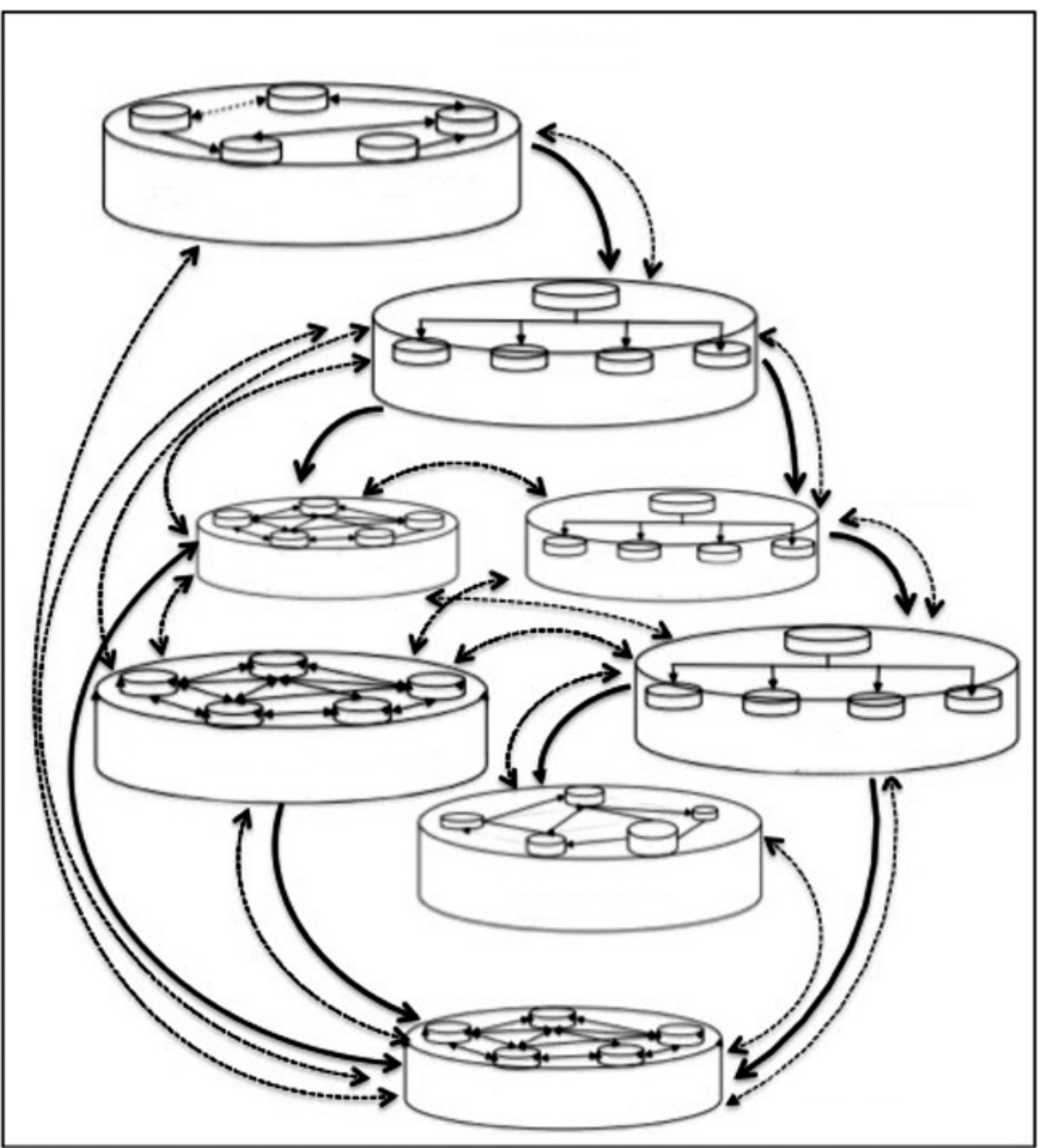

Fuente: elaboración propia con base en Berkes (2007).

organizativas para el desarrollo de otras actividades productivas.

La lógica colectiva también se hace presente por medio de la participación de organizaciones no gubernamentales (ONG). La gestión estatal de estas áreas se encuentra apoyada por la presencia de diferentes

ONG internacionales ${ }^{5}$ y nacionales ${ }^{6}$, la cuales actúan de manera conjunta con los actores locales para presionar al gobierno respecto de la elaboración de marcos normativos para el desarrollo de actividades sustentables (Damoulin \& Rodary, 2005; Dumoulin, 2007). No obstante, al
5 Por ejemplo, The Nature Conservancy (TNC), World Wild Found (WWF) $y$ Conservation International (CI).

${ }^{6}$ Pronatura, Biocenosis o Amigos de Sian Ka'an.

tersitarias 40 
${ }^{7}$ En México, la World Wildlife Fund (WWF) tiene importantes Alianzas con la Fundación Carlos Slim, con la empresa de telefonía Telcely con la Fundación Gonzalo Rio Arronte, I.A.P., que están relacionadas con dos de los empresarios más importantes del pais.

\section{territarias 40}

presentarse ante la sociedad como verdaderas multinacionales de la conservación, ponen en entre dicho la autenticidad de sus objetivos colectivos ${ }^{7}$.

Finalmente, también se reconocen aquellos actores orientados por una lógica pública, en el caso de las ANP mexicanas, el Estado - por medio de sus diferentes niveles de gobierno- es el agente protagónico en la gestión del interés colectivo en estas áreas. Las instituciones gubernamentales encargadas de su administración, generalmente, lo hacen bajo un enfoque centralista y sectorial (Caravaca \& González, 2009, Brenner, 2010, 2014).

Con esta lógica de acción también se encuentran vinculadas las universidades, centros de investigación y de transferencia tecnológica. Su presencia resulta importante cuando se trata de la búsqueda de alternativas a las diversas demandas relacionadas con el desarrollo socioeconómico de estos territorios. El "mapeo", como parte de proceso metodológico para el diagnóstico de la estructura de las relaciones sociales, permite identificar a los actores en sus múltiples niveles de acción; es necesario prestar atención a la diversidad de agentes que participan en estos territorios para evitar considerarlos como entidades homogéneas y autocontenidas (Nygren, 2005).

De manera general, estas son las clasificaciones que en un ANP pueden contribuir a visualizar el escenario social, por supuesto, la diversidad de actores también está en función de la categoría en la que esté considerada el área de estudio, los intereses políticos y económicos vinculados al territorio en cuestión.

\subsection{El ámbito de las relaciones micro-meso: el capital social cognitivo y relacional}

Si bien, han surgido un conjunto de enfoques y conceptos en torno al capital social (Coleman, 1990; Putnam, 1993; Bourdieu, 1995; Fukuyama, 1999), la diferencia entre cada uno de estos radica, entre otras cosas, en el nivel de la estructura en el que se focalizan los esfuerzos de análisis, es decir, en los vínculos que guarda un actor con otro o en las relaciones entre los actores en una colectividad e incluso si se abordan las formas de organización adoptadas por una población para hacer frente a sus problemas de producción, reproducción, regulación, entre otros aspectos (Turner, 2000; Adler \& Kwon, 2000, 2002).

La valorización actual de los recursos naturales coloca, en este caso, a las ANP en el centro de un cúmulo de relaciones a nivel local y global y de tensiones por la producción y comercialización de los recursos funcionales a la dinámica económica mundial. Lo anterior supone la presencia de una amplia diversidad de actores sociales que se manifiestan a diferentes escalas de la estructura de las relaciones, es decir, que se mantienen y rebasan el ámbito comunitario/local mediante la construcción de relaciones horizontales y verticales de coordinación, comunicación, negociación e incluso de confrontación. 
En este sentido, se retoma nuevamente la idea tripartita de lo micro-meso-macro, como los tres niveles de análisis desde los cuales se puede encarar el estudio de la estructura de las relaciones en la gestión de estos ecosistemas (Turner, 2000). En el caso de las ANP, estos niveles permiten indagar en las relaciones informales e interpersonales que se presentan en el ámbito comunitario y extracomunitario, pero también en las relaciones jerárquicas donde existe una distribución desigual de poder entre los miembros y, en las relaciones formalizadas e institucionalizadas. En resumen, los tres niveles constituyen espacios de relaciones sociales donde se gestan las estructuras y los mecanismos de gobernanza.

En este trabajo se propone que el estudio de las relaciones a nivel micro debe llevarse a cabo desde el enfoque cognitivo y relacional del Cs, es decir, por medio de los recursos sociales que emanan de las percepciones individuales acerca del grado interpersonal de confianza y de las normas de reciprocidad entre los individuos. En este nivel, el CS se constituye como un elemento esencial en la cohesión social inter y extracomunitaria constituida por aquellos procesos mentales, ideologías y formas culturales que permiten las relaciones basadas en normas compartidas, mecanismos de cooperación, confianza, reciprocidad que se establecen entre los involucrados.

Ambas dimensiones del cs son propicias para indagar sobre la construcción de vínculos entre individuos, grupos primarios que son retroalimentados por un historial de interacciones en el tiempo y estimulados por activos como la confianza y reciprocidad (Putnam,1993; Fukuyama, 1999), obligaciones y expectativas (Putnam, 1993); normas y sanciones (Coleman, 1990) y otros como la identidad o la participación.

Estas facetas del Cs actuan como la columna vertebral que da soporte a la dinámica relacional pero a nivel local. En el caso de las ANP mexicanas, se sabe que las comunidades campesinas e indígenas son pieza angular en relación con las formas de aprovechamiento de los recursos, ya que son las usufructurarias históricas (generalmente), legales y directas de estos territorios (Botelho, 2008; Moyano, 2001).

Ambas formas de CS se encuentran ligadas a "los valores éticos y sociales de los individuos que determinan cómo se relacionan unos con otros" (Foronda \& Galindo, 2012, p. 48); por tanto, son componentes vinculados con los procesos mentales y culturales que llevan a los integrantes de una comunidad a actuar con base en su "juicio moral”. En este sentido, son tipos de capital que sientan las bases para la construcción de estructuras relacionales fuertes; no obstante, carecen de potencial para inducir a los individuos a actuar de manera colectiva; si bien, establecen en ellos una predisposición, permanecen en el plano de sus emociones y estados psicológicos sin transitar al plano de la acción colectiva $^{8}$, son recursos intrínsecos al individuo pero no una acción per se.
${ }^{8}$ Desde las teorias de la elección racional, el CS es visto como un recurso que emerge de la interacción entre agentes racionales destacando su dimensión contractual, en el sentido de que los individuos cooperan entre si solo porque consideran que es bueno para sus intereses hacerlo, por tanto, es un comportamiento racional. En cambio, el planteamiento culturalista (del cual emana enfoque cognitivo y relacional) $v i$ sualiza al Cs desde las relaciones sociales y su potencial para facilitar a los individuos determinados tipos de acciones colectivas (Moyano, 2008).

tersitarias 40 41 
${ }^{9}$ De acuerdo con Herreros (2001), estos recursos pueden consistir en la adquisición de información, en obligaciones derivadas de acciones de reciprocidad, en sistemas de confianza o en el sugimiento de normas sociales cooperativas, entre otros recursos.

\section{territarias 40}

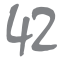

Es necesario que en el seno de la gobernanza ambiental se encuentre la vinculación de los actores sociales a distintas escalas que lleven a la formulación de decisiones conjuntas para el adecuado manejo y conservación de las ANP. La gobernanza ambiental en términos de los recursos sociales requiere del fortalecimiento de las redes e instituciones (formales e informales, horizontales y verticales) que dan cauce a la acción social. Siendo así, deben ser visualizadas aquellas formas de cs que faciliten el diagnóstico de las relaciones, los vínculos y las redes para la conformación de organizaciones sociales con capacidad para el intercambio intracomunitario, $y$ con grupos u organizaciones de niveles y territorialidades múltiples.

\subsection{El capital social estructural (CSE): la estructura de las relaciones}

Se reconoce que los actores sociales en una ANP cuentan en sí mismos con recursos (información, experiencia, económicos, sociales, entre otros) que incentivan las relaciones bajo condiciones socioestructurales similares. No obstante, la complejidad social de territorios como las ANP mexicanas exige que el análisis de la dinámica relacional vaya más allá de las relaciones entre individuos y organizaciones locales, con la finalidad de comprender también la red de relaciones sinérgicas a nivel vertical.

La definición estructural de capital social se deriva de los trabajos de Bourdieu (1995) y Coleman (1990), quienes lo conceptualizan como el conjunto de recursos disponibles ${ }^{9}$ para el individuo derivados de su participación en redes sociales, que facilitan la consecución de objetivos colectivos. A diferencia del enfoque cognitivo y relacional (Putnam, 1993; Fukuyama, 1999), el estructural se centra en el capital social que se genera y se acumula en las redes sociales entre grupos en iguales o disímiles posiciones económicas o políticas (Sabatini, 2009; Rostila, 2010). En el CSE, las relaciones son vistas en términos de enlaces y como estrategias de comunicación, transmisión de información y aprendizaje, pero también representan estructuras de poder orientadas hacia la institucionalización de las relaciones grupales.

A manera de referencia empírica, las ANP mexicanas se caracterizan por el alto involucramiento de las instituciones gubernamentales —debido a la asignación de recursos financieros o humanos, ejecución, coordinación y control de acciones-, las cuales regularmente mantienen una política tradicional de conservación que ha mostrado ser poco eficaz (Barkin, 2000; Zizumbo, Cruz, \& Vilchis, 2012; Zizumbo, 2013). En este caso, el CSE se convierte en un importante instrumento para el diagnóstico de la sinergia organizacional, es decir, de las relaciones de cooperación, negociación y de confrontación de intereses entre instituciones $\mathrm{y}$, entre estas y otros grupos de actores sociales de distintos niveles (micro-meso-macro).

La gobernanza ambiental es el espacio en el cual se construyen las leyes, las políticas, las regulaciones, los procedimientos formales y los códigos de conducta que 
inciden en la conservación y el aprovechamiento de los recursos en estos ecosistemas. Por tanto, la gobernanza se relaciona con quiénes y cómo se toman las decisiones, pero aún más con las características de los vínculos sociales, que facilitan la coordinación y la cooperación para el beneficio mutuo de los actores sociales. De acuerdo con Bowles \& Gintis (2002), estas características incluyen redes, normas sociales de reciprocidad y confianza, las cuales, si se usan de manera positiva, incentivan la acción colectiva para lograr un cambio del modo de gobernanza jerárquica a uno basado en redes de relaciones horizontales.

De manera general, las redes cumplen un rol particular, si bien el CSE no es sinónimo de redes sociales, sin estas no puede generarse este tipo de CS. El estudio del CSE en las ANP puede contribuir a la comprensión formal de las redes de relaciones y de los recursos (confianza, solidaridad, conocimiento, información, entre otros) que de ellas emanan. Este tipo de capital da cuenta de los enlaces efectivos entre individuos (bonding social capital) de una misma comunidad; no obstante, su potencial radica en su capacidad explicativa en torno a los vínculos extralocales, es decir, entre comunidades y grupos conformados a nivel regional (brindging social capital) y entre estos y los organismos gubernamentales y no gubernamentales a nivel nacional e incluso internacional (linking social capital) (Grafton, 2005).

En términos de gobernanza ambiental, este tipo de capital no solo puede evidenciar las relaciones efectivas ${ }^{10}$ entre diferentes actores sociales y a distintos niveles de la estructura social; sino tambien cumple un rol importante en la comprensión de las relaciones de conflicto, cooperación, alianza y de las situaciones que las provocan. Por tanto, el CSE es una herramienta metodológica que permite dimensionar el esquema relacional de un territorio en términos formales, identificando pautas de interacción, influencia, subordinación, accesibilidad y conectividad entre los actores que componen una red de relaciones (nivel horizontal) y, entre estos con actores de niveles superiores (nivel vertical).

Para el estudio del CSE se han propuesto algunas características formales significativas que pueden desglosarse en posibles variables operativas y que son susceptibles de contraste empírico. Por ejemplo, la intensidad relacional, la cual encuentra su expresión en la direccionalidad de las relaciones, la frecuencia y los tipos de lazos que se establecen entre los actores sociales.

La direccionalidad es pertinente en la medida que indica si las relaciones en la red son de reciprocidad, de autoridad/ poder, conflicto o de tipo instrumental (Natera, 2001; Granovetter, 1982); esto da la pauta para conocer la influencia que ejercen unos actores sobre otros acerca del tipo de aprovechamiento que se hace de los recursos naturales en estas áreas. Salvo muy escasas excepciones, la mayoría de las ANP se han convertido en espacios altamente conflictivos donde la direccionalidad de los vínculos entre los actores públicos y privados denotan la existencia de relaciones paternalistas y clientelares
${ }^{10}$ Cuando se hace referencia a relaciones efectivas, estas no solo pueden ser de cooperación sino también de transferencia de conocimiento, de información, de conflicto, etc. tersitarios 40

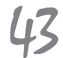


(Paz, 2008; Brenner, 2010), más que en procesos autogestivos y democráticos.

En el diagnóstico del CSE, la centralidad es también un indicador importante debido a que permite identificar aquellos actores que están bien "conectados" en su entorno local y extralocal y que, por tanto, influyen en las decisiones en torno al aprovechamiento de las ANP. En este sentido, la sociedad debe ser vista como un mercado en donde la gente intercambia toda clase de ideas y bienes en la búsqueda de sus intereses y en donde los actores sociales, que son los productores y consumidores de cierto tipo de capitales, establecen relaciones que colocan a algunos como los actores dominantes en la escena de la estructura social (Coleman, 1990; Bourdieu \& Wacquant, 1992; Putnam, 1993). Siguiendo lo anterior, la centralidad es indicativo del grado de disparidad en los vínculos, la desigual distribución del poder entre los actores y la ventaja o desventaja que tienen algunos sobre otros en la esfera del aprovechamiento de los recursos.

En México, la centralidad de los organismos gubernamentales en la toma de decisiones en torno a la gestión turística de las ANP ha limitado el aprovechamiento de estos ecosistemas por parte de otros actores sociales. La CONANP ha creado algunos espacios de participación, tales como los consejos técnicos asesores, los subconsejos, los comités promotores, entre otros que, aparentemente, actuan como órganos de negociación de intereses entre la sociedad y el Estado. No obstante, algunos estudios de caso demuestran que desde su creación estos consejos surgen como órganos reducidos a la mera posibilidad de emitir "sugerencias", por tanto, dan lugar a procesos de exclusión interna que refuerzan las inequidades al interior de las ANP (Peterson, 2011; Durand, Figueroa, \& Trench, 2012).

La perspectiva estructural permite analizar el capital social en términos de modelos de relaciones sociales que vinculan a los actores sociales individuales y colectivos por medio de redes que evidencian el intercambio de formas de CSE tales como la información, conocimiento, las obligaciones de reciprocidad, confianza, entre otros. Siguiendo en el plano metodológico, dicho enfoque implica la representación sistemática de los vínculos entre actores como redes; por tanto, utiliza las herramientas derivadas de la teoría de los grafos (direccionalidad, centralidad, etc.) para comprender los patrones de relación de los vínculos. La metodología exige la aplicación de diferentes técnicas de investigación cualitativa (entrevistas a profundidad, grupos de enfoque, historias de vida, entre otros) y cuantitativa (cuestionario) que generen los datos primarios que permiten esbozar un primer acercamiento a la estructura de las relaciones en territorios socialmente tan complejos como las ANP (figura 3 ).

Este enfoque desecha la importancia de las relaciones en términos de calidad, pero permite diagnosticar el capital social en función de la conectividad entre los actores sociales, su accesibilidad a la información, a los recursos y al poder y, a la posición 


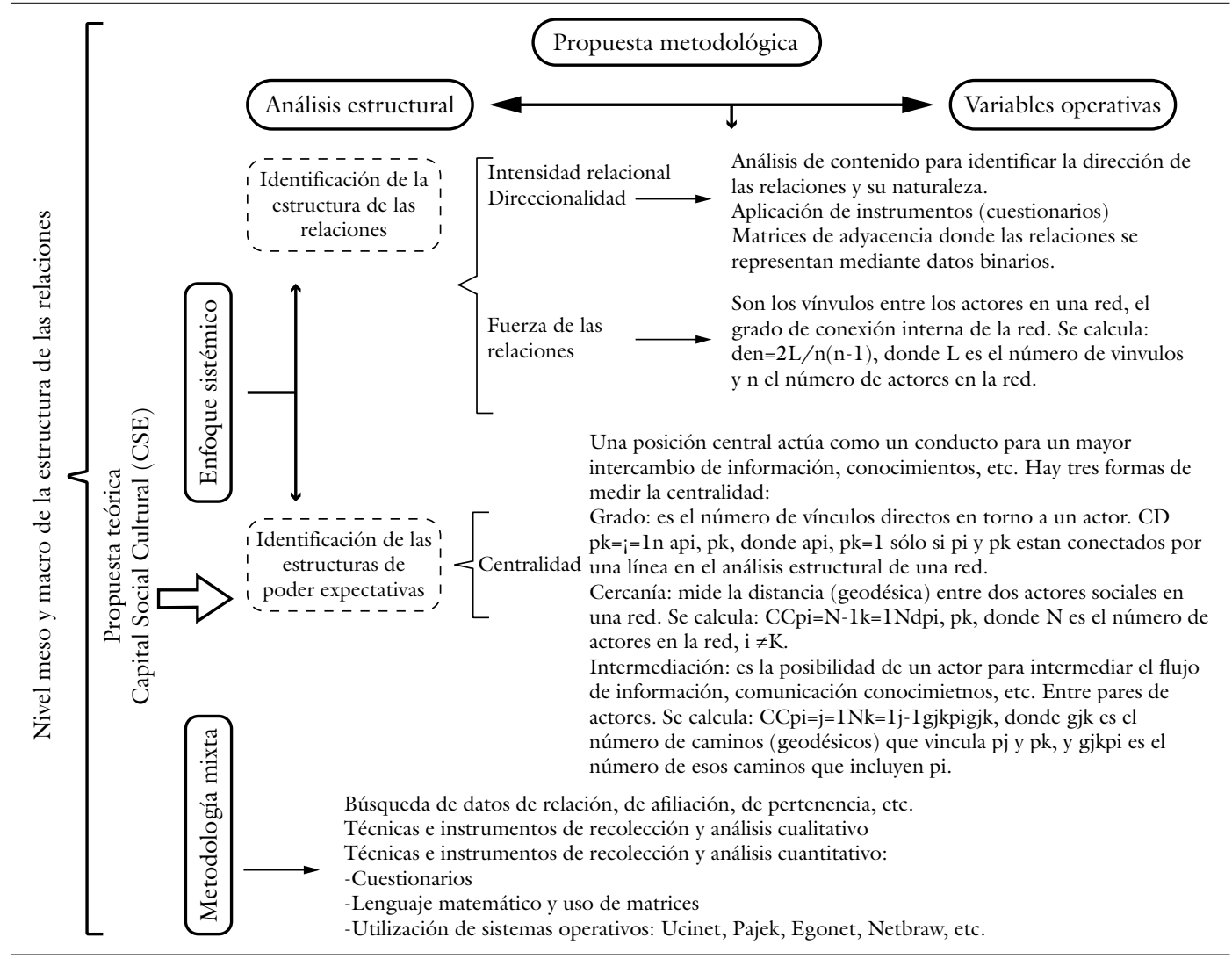

Fuente: elaboración propia a partir de Wasserman \& Faust (1994), Wellman (2000).

que este tipo de cs concede a los actores en la estructura de las relaciones para la consecución de los objetivos individuales pero, sobre todo, colectivos.

\section{Conclusiones}

A lo largo de esta reflexión, se presenta el capital social como un proceso interactivo que facilita la cooperación individual y de grupo, así como la organización de insti- tuciones a escalas diferentes; por tanto, en este trabajo se plantea la existencia necesaria de capital social en sus diferentes facetas como prerrequisito para la conformación de estructuras de gobernanza ambiental a favor de la gestión descentralizada de las áreas de protección, particularmente, de las ANP mexicanas.

Este trabajo propone el estudio de la dinámica relacional en dichos territorios catalogados como socialmente complejos, 
debido a la diversidad de actores sociales cuyos intereses convergen en estas áreas de conservación. Se retoma, particularmente el estudio del CSE, por su capacidad explicativa en relación con la naturaleza de las relaciones entre los actores de diferentes niveles (micro-meso-macro) de la estructura social. Es considerado como una de las principales herramientas para el diagnóstico de la estructura de las relaciones en la gobernanza ambiental de las ANP, debido a que permite mirar la complejidad del territorio en términos sociales; es decir, reconoce la interacción de múltiples agentes, pero no necesariamente en términos lineales y positivos. Por tanto, este tipo de capital social permite describir dichos territorios como una arena de relaciones de conflicto, de poder, de resistencia, de subordinación, de cooperación y de intercambio de recursos (información, recursos económicos, innovación, entro otros).

El estudio del CSE se apoya en otras herramientas metodológicas de tipo cualitativo, pero, sobre todo, cuantitativo (enfoque de redes y sus herramientas metodológicas) a partir de las cuales se genera información valiosa respecto a los patrones de vinculación entre los actores sociales que, en definitiva, se apoyan en el estudio del territorio como espacios de múltiples demandas, objetivos y valoraciones.

La reflexión se torna importante en la medida que las ANP requieren, de manera urgente, que se genere un cambio en las formas de gestionar los recursos: de la gobernabilidad a otra fundamentada en procesos de gobernanza ambiental.
Particularmente, en las ANP mexicanas se hace cada vez más necesaria la implementación de formas de gobernanza ambiental más eficaces y socialmente más equilibradas; por tanto, es indispensable la generación de conocimiento del territorio en términos sociales, culturales y políticos a fin de alcanzar análisis más integrales.

Retomando a De Castro, Hogenboom \& Baud (2015), "las interacciones sociales son el motor de la gobernanza ambiental" (p. 27). En la medida que las ANP sean visualizadas como espacios políticos de relaciones dinámicas y variadas, podrán generarse ciertas adaptaciones institucionales - tales como el re-escalamiento de la gobernanza ambiental al nivel local- que permitan transformar las estructuras de gobernanza ambiental para contrarrestar los procesos predominantes de "cooperación" y "participación" verticalistas existentes en la mayoría de las ANP mexicanas.

\section{Referencias}

Abbott, K., Green, J., \& Keohane, R. (2016). Organizational Ecology and Institucional Change in Global Governance. International Organization, 70(2), 247-277.

Adler, P., \& Kwon, S. W. (2000). Social capital: The good, the bad and the ugly. En E. Lesser (ed.), Knowledge and social capital: Foundations and applications (pp. 89-115). Boston: ButterworthHeinemann.

Adler, P., \& Kwon, S. W. (2002). Social Capital Prospects for a New Concept. 
The Academy of Management Review, 17-40.

Alonso, O. (2004). La lógica de los actores y el desarrollo local. Revista Pilquen, $1-12$.

Arriola, V., Estrada, E., Ortega, A., Pérez, R., \& Gijón, A. (2014). Deterioro en áreas naturales protegidas del centro de México y del Eje Neovolcánico Transversal. Investigación y Ciencia, 60, 37-49.

Azuela, A., \& Mussetta, P. (2009). Algo más que el ambiente. Conflictos sociales en tres áreas naturales protegidas de México. Revista de Ciencias Sociales, 16, 191-215.

Barkin, D. (2000). The economic impacts of ecoturism: Conflicts and solutions in highland Mexico. En P. Godde, M. Price, \& F. Zimmermann (Coords.), Tourism and development in mountain areas (pp. 157-172). Londres: CAB International.

Beritelli, P. (2011). Cooperation among prominent actors in a tourist destination. Annals of Tourism Research, 607-629.

Berkes, F. (2007). Community-based conservation in a globalized world. Proceedings of the National Academy of Sciences of the United States of America, 104(39), 15188-15193.

Blicharska, M., Orlikowska, E., Roberge, J. M., \& Jurczak, M. (2016). Contribution of social science to large biodiversity conservation: A review of research about the Natura 2000 network. Biological Conservation, 199, 110-122.

Botelho, A. (2008). El protagonismo de las comunidades rurales como fundamento de la cogestión adaptativa incluyente de los recursos naturales en un territorio. (Tesis de Maestría, Centro Agronómico Tropical de Investigación y Enseñanza (CATIE), Turrialba, Costa Rica).

Bourdieu, P. (1995). Respuestas por una antropología reflexiva. México: Editorial Grijalbo.

Bourdieu, P., \& Wacquant, L. (1992). An invitation to Reflexive Sociology. Chicago, USA: The University Chicago Press. Bowles, S., \& Gintis, H. (2002). Social Capital and Community Governance. The Economic Journal, 112(483), 1-24.

Brenner, L. (2010). Gobernanza ambiental, actores sociales y conflictos en las Áreas Naturales Protegidas. Revista Mexicana de Sociología, 283-310.

Brenner, L., \& De la Vega, A. (2014). La gobernanza participativa de áreas naturales protegidas. El caso de la Reserva de la Biosfera El Vizcaíno. Región y Sociedad, 26(59), 183-213.

Caravaca, I., \& González, G. (2009). Las redes de colaboración como base del desarrollo territorial. Scripta Nova, Revista de Electrónica de Geografía y Ciencias Sociales, 13(289). Recuperado de http://www.ub.edu/geocrit/sn/ sn-289.htm

Coleman, J. (1990). Foundations of social theory. Cambridge: Belknap Press of Harvard University Press.

Comisión Nacional de Áreas Naturales Protegidas (26 de abril de 2018). Secretaría de Medio Ambiente y Recursos Naturales. Recuperado de www.conanp.gob. $\mathrm{mx}$ /que_hacemos/ territarios 40 47 
Comisión Nacional de Áreas Naturales Protegidas (2017). Áreas Naturales Protegidas Federales de México. Mayo 2017. México: Comisión de Áreas Naturales Protegidas.

Cruz, E., Zizumbo, L., Monterroso, N., \& Quintanilla. A. (2013). La confrontación social por el espacio costero: la configuración de paisajes turísticos en Puerto Morelos, Quintana Roo. Región $y$ sociedad, 25(56), 127-160.

Cruz, E., Harald, A., \& Briones, A. (2016). Collaborative Networks and Tourism Management of Peri-Urban Forests. International Development Journal of Sustainable Development and Planning, 11(2), 172-181.

Damoulin, D., \& Rodary, E. (2005). Les ONG et le secteur mondialise de la conservation. En C. Aubertin (Ed.), Représenter la nature. ONG et biodiversité (pp. 59-98). Paris: Presse de l'IRD.

De Castro, F., Hogenboom, B., \& Baud, M. (2015). Gobernanza Ambiental en América Latina en la Encrucijada. Moviéndose entre múltiples imágenes, interacciones e instituciones. En F. de Castro, B. Hogenboom \& M. Baud (Coords.), Gobernanza ambiental en América Latina (pp. 13-38). Buenos Aires: CLACSO.

Dumoulin, D. (2007). Las políticas de las ANP (Áreas Naturales Protegidas) como laboratorio para los esquemas públicoprivados. Una interpretación a partir del Fondo Mexicano para la Conservación territarias 40 48 de la Naturaleza. En G. Fontaine, G. Van Vliet, \& R. Pasquis (eds.), Viajes en los terruños de la gobernabilidad en las políticas ambientales en América Latina (pp. 57-78). Quito: FLACSO-Ecuador.

Durand, L. (2014). ¿'Todos ganan? Neoliberalismo, naturaleza y conservación en México. Sociológica, 29(82), 183-223. Durand, L., Figueroa, F., \& Trench, T. (2012). Inclusión, exclusión y estrategias de participación en áreas protegidas de la Selva Lancandona, Chiapas. En L. Durand, F. Figueroa, \& M. Guzmán (Eds.), La naturaleza en contexto. Hacia una ecología politica mexicana (pp. 237-267). México: Centro de Investigaciones Interdisciplinarias en Ciencias y Humanidades-Centro Regional de Investigaciones Multidisciplinarias-El Colegio de San Luis.

Esquivel, S., Cruz, G., Cadena, C., \& Zizumbo, L. (2014). El turismo como instrumento de política ambiental en el Santuario de la Mariposa Monarca El Rosario. Economía, sociedad y territorio, 14(44), 141-174.

Foronda, C., \& Galindo, L. (2012). Argumentación relativa a la confianza territorial. Claves sobre capital social. Cuadernos de Desarrollo Rural, 9(68), 41-63.

Fukuyama, F. (1999). Social Capital and Civil Society. Virginia: George Manson University.

Grafton, Q. (2005). Social capital and fisheries governance. Ocean \& Coastal Mangement, 48(9), 753-766.

Granovetter, M. (1982). The strength of weak ties. A. En P. Marsden, \& N. Li (eds.), Social structure and network 
analysis (pp. 201-233). Beverly Hills, USA: Sage Publications.

Grimble, R., \& Wellard, K. (1999). Stakeholder methologies in natural resource management: a review of principles, contexts, experiences and opportunities. Agricultural Systems, 55(2), 173-193.

Herreros, F. (2001). ¿Son las relaciones sociales una fuente de recursos? Una definición del capital social. Papers, Revista de Sociología de la Universidad de Barcelona, 67, 129-148.

Leeuwis, C., \& Van den Ban, A. (2004). Communication for Rural Innovation: Rethinking Agricultural Extension. Oxford: Wiley-Blackwell.

Lugo, D. (2011). Análisis de redes sociales en el mundo rural: guía inicial. Revista de Estudios Sociales, 129-142.

Marín, A., Gelcich, S., Castilla, J. C., \& Berkes, F. (2012). Exploring Social Capital in Chile's. (Tesis de Maestría. Natural Resources Institute, University of Manitoba, Winnipeg, Canadá).

Melucci, A. (1996). Challenging codes. United Kingdom: University Press Cambridge.

Moyano, E. (2001). El concepto de capital social y su utilidad para el análisis de las dinámicas del desarrollo. Revista de Fomento Social, 56, 35-63.

Moyano, E. (2008). Capital social y acción colectiva en el sector agrario. RES. Revista Española de Sociología, 10, 15-37.

Natera, A. (2001). El liderazgo político en la sociedad democrática. Revista de Estudios Politicos, 385-421.
Natera, A. (2005). La gobernanza como modo emergente de gobierno y gestión pública. Gestión y Análisis de Politicas Públicas, (33-34), 53-65.

Nenadovic, M., \& Epstein, G. (2016). The relationship of social capital and fishers's participation in multi-level governance arrangements. Environmental Science \& Policy, 61, 77-86.

Nunkoo, R. (2017). Governance and sustainable tourism: What is the role of trust, power and social capital? Journal of Destination Marketing \& Management, 6(4), 277-285.

Nygren, A. (2005). Community-based Forest Management within the Context of Institutional Descentralization in Honduras. World Development, 33(4), 639-655.

Ojo, A., \& Mellouli, S. (2016). Deploying governance networks for societal challenges. Government Information Quarterly [In press].

Ostrom, E. (1990). Governing the commons: The Evolution of Institutions for Collective Action. Cambridge: Cambridge University Press.

Paavola, J., \& Adger, N. (2005). Institutional ecological economics. Ecological Economics, 53(3), 353-368.

Paré, L., \& Lazos, E. (2003). Escuela rural y organización comunitaria: Instituciones locales para el desarrollo y el manejo ambiental. México: UNAM-IIS-Plaza y Valdés.

Paz, M. F. (2008). De áreas naturales protegidas y participación: Convergencias

territorias 40

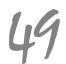


y divergencias en la construcción del interés público. Nueva Antropología, Revista de Ciencias Sociales, 68, 51-74.

Pérez, C., Zizumbo, L., \& Monterroso, N. (2009). Turismo e identidad de resistencia; La oposición local a proyectos turísticos en el Parque Nacional Nevado de Toluca, México. Estudios y Perspectivas en Turismo, 18(1), 36-52.

Peterson, N. (2011). Excluding to Include: (Non) Participation in Mexican Natural Resource Management. Agriculture and Human Values, 99-107.

Putnam, R. (1993). Making Democracy Work: Civic Traditions in Modern Italy. Princeton: Princeton University Press. Rabotnikof, N. (2008). Lo público hoy: lugares, lógicas y expectativas. Íconos, Revista de Ciencias Sociales, 37-48.

Rist, S., Chimdambaranathan, M., Escobar, C., Wiesmann, U., \& Zimmermann, A. (2007). Moving from sustainable management to sustainable governance of natural resources: The role of social learning processes in rural India, Bolivia and Mali. Journal of Rural Studies, 23, 23-37.

Rostila, M. (2010). The Facets of Social Capital. Journal of the Theory of Social Behaviour, 308-326.

Sabatini, F. (2009). Social capital as social networks: A new framework for measurement and a empirical anlalysis of its determinants and consequences. The Journal of Socio-Economics, 429-442.

Schejtman, A., \& Berdegué, J. (2004). Desarrollo territorial rural. Debates y temas rurales. Santiago: Rimisp/Centro Latinoamericano para el Desarrollo.

Selig, E., \& Bruno J. (2010). A Global Analysis of the Effectiveness of Marine Protected Areas in Preventing Coral Loss. PLos ONE, 5(2), 1-12.

Stoll-Kleemann, S., Bender, S., Berghofer, A., Bertzky, M., Fritz-Vietta, N., Schliep, R., et al. (2006). Linking Governance and Management Perspectives with Conservation Success in Protected Areas and Biosphere Reserves. Berlin: GoBi Research Group.

Tejeda, C. (2014). Conservación de la Biodiversidad y Comunidades Locales: Conflictos en áreas Naturales Protegidas de la Selva Lacandona, Chiapas, México. Canadian Journal of Latin American and Caribbean Studies, 34(68), 57-88.

Toledo, V. (2005). Repensar la conservación: ¿áreas naturales protegidas o estrategia bioregional? Gaceta Ecológica, 77, 67-83.

Triyanti, A., Bavink, M., Gupta, J., \& Marfai, M. (2017). Social capital, interactive governance and coastal protection: The effectiveness of mangrove ecosystembased strategies in promoting inclusive development in Demak, Indonesia. Ocean \& Coastal Mangement, 150(1), 3-11.

Turner, J. (2000). The Formation of Social Capital. En P. Dasgupta \& I. Seralgeldin (Coord.), Social Capital. A Multifaceted Perspective (pp. 94-146). Washington: The World Bank.

Valencia, J. (1990). La lógica de la acción colectiva: tres modelos de análisis de la 
participación política no insitucional. Revista de Psicología Social, 185-214.

Wasserman, S., \& Faust, K. (1994). Social Network Analysis: Methods and Applications. England: University Press.

Wellman, B. (2000). El análisis estructural: del método y la metáfora a la teoría y la sustancia. Politica y Sociedad, 33, 11-40.
Zizumbo, L. (2013). Las paradojas del desarrollo local y el turismo. México: Porrúa. Zizumbo, L., Cruz, E., \& Vilchis A. (2012). Acción social y Áreas Naturales Protegidas: Refuncionalización de Recursos en Puerto Morelos, Quintana Roo, México. Revista Rosa dos Ventos, 4(2), 192-207. 
